\title{
Comparison of random regression and repeatability models to predict breeding values from test-day records of Norwegian goats
}

\author{
S. Andonov, ${ }^{* 1}$ J. Ødegård,† M. Svendsen, $\ddagger$ T. Ådnøy,† M. Vegara,§ and G. Klemetsdal† \\ ${ }^{*}$ Faculty of Agricultural Sciences and Food, University Ss Cyril and Methodius, PO Box 297, 1000 Skopje, Macedonia \\ †Department of Animal and Aquacultural Sciences, Norwegian University of Life Sciences (UMB), PO Box 5003, N-1432 Ås, Norway \\ $\ddagger$ Geno, Breeding and Al Association, Norwegian University of Life Sciences (UMB), PO Box 5003, N-1432 Ås, Norway \\ §Department of International Environment and Development Studies, NORAGRIC, UMB, PO Box 5003, N-1432 Ås, Norway
}

\begin{abstract}
One aim of the research was to challenge a previously selected repeatability model with 2 other repeatability models. The main aim, however, was to evaluate random regression models based on the repeatability model with lowest mean-squared error of prediction, using Legendre polynomials up to third order for both animal additive genetic and permanent environmental effects. The random regression and repeatability models were compared for model fit (using likelihood-ratio testing, Akaike information criterion, and the Bayesian information criterion) and the models' mean-squared errors of prediction, and by cross-validation. Cross-validation was carried out by correlating excluded observations in one data set with the animals' breeding values as predicted from the pedigree only in the remaining data, and vice versa (splitting proportion: 0.492). The data was from primiparous goats in 2 closely tied buck circles (17 flocks) in Norway, with 11,438 records for daily milk yield and 5,686 to 5,896 records for content traits (fat, protein, and lactose percentages). A simple pattern was revealed; for daily milk yield with about 5 records per animal in first lactation, a second-order random regression model should be chosen, whereas for content traits that had only about 3 observations per goat, a first-order polynomial was preferred. The likelihood-ratio test, Akaike information criterion, and mean-squared error of prediction favored more complex models, although the results from the latter and the Bayesian information criterion were in the direction of those obtained with cross-validation. As the correlation from cross-validation was largest with random regression, genetic merit was predicted more accurate with random regression models than with the repeatability model.
\end{abstract}

Key words: milk trait, model fit, cross-validation

\footnotetext{
Received July 5, 2012.

Accepted November 30, 2012.

${ }^{1}$ Corresponding author: sandonov@zf.ukim.edu.mk
}

\section{INTRODUCTION}

In Norway, a cooperative genetic improvement scheme for buck circles, covering $42 \%$ of the national goat population, has been well established (Nævdal et al., 2005). In a buck circle and each year, the participating goat herds (average: 9) typically mate with selected young bucks (5). When their daughters came into production, the sires (2) with the best predicted breeding values were mated to the (30\%) elite goats in the circles (Ådnøy et al., 2000). Circles were tied together by use of common elite bucks, either sold live and used in natural mating (after having been used 1 yr in the buck circles where they were proven) or as frozen semen from AI bucks, used across buck circles. In recent years, the number and size of buck circles has been reduced due to a sanitation program for caprine arthritis-encephalitis virus (CAEV), which requires no contact of live animals across herds.

The dairy recording system requires at least 5 testday records per flock and year for daily milk yield (DMY) of which at least 3 are sampled for fat (FC), protein $(\mathbf{P C})$, and lactose content $(\mathbf{L C})$, both in first and second lactation. Since 1994, the animals have been genetically evaluated by a multitrait repeatability (REP) model, fitting the lactation curve with a fixed Ali-Schaeffer regression (ASR; Ali and Schaeffer, 1987), nested within year-season of kidding (YS), regions, and lactation numbers, and with random effects of flock test day (FTD), animal, and permanent environment (Adnøy et al., 2000).

Andonov et al. (2007) examined alternatives to the above model (single trait, within region, and in first lactation) and suggested the inclusion of a fixed structure with year (modeled as YS) and a description of the lactation curve by fixed effects of DIM grouped in 3-d periods (DIM3). Here, one aim was to challenge the suggested model with 2 alternative REP specifications: one in which the lactation curve was modeled with ASR, across YS, and another with DIM3, that had flock year (FY) as fixed and FTD as random effects; the latter was hypothesized to increase genetic vari- 
ance (Andonov et al., 2007). However, the main aim of the current study was to evaluate random regression (RR) models based on the REP model with lowest mean-squared error of prediction, using Legendre polynomials (Kirkpatrick and Heckman, 1989) of different orders for modeling both animal additive genetic and permanent environmental effects, also to estimate genetic parameters for DMY, FC, PC, and LC. Models were compared through model fit, mean-squared error of prediction, and predictive ability by cross-validation.

\section{MATERIALS AND METHODS}

\section{Data}

The data were the same as used in the study of Andonov et al. (2007), for primiparous goats in 2 closely tied buck circles ( 7 and 10 flocks, respectively, with 24 to 350 animals per flock over the study period) in one region of Norway (Hedmark). Data was from years 1988 to 2003, with records for DMY ( $\mathrm{n}=11,438$ from 2,371 goats), $\mathrm{FC}$ and $\mathrm{PC}$ ( $\mathrm{n}=5,896$ from 2,215 goats), and $\mathrm{LC}(\mathrm{n}=5,686$ from 2,111 goats). Records from 5 to 305 DIM were kept when ranging 0.1 to $9.9 \mathrm{~kg}$ per day for DMY. Fat content, PC, and LC values between 1 and $9 \%$ were accepted. Each year, 3 seasons of kidding were used (December to February, March to May, and June to November, with 1 to 464 records for content traits), in accordance with the current practice in genetic evaluation.

The pedigree data involved all animals with records and their ancestors traced back as far as possible. The pedigree data contained 5,511 animals $(75.96 \%$ had both parents known); of these, 421 were sires and 5,090 were dams, with 261 animals defining the base population. For animals with DMY records, the average number of generations known [the complete generation equivalent, according to Boichard et al. (1997)] was 3.27.

\section{REP Models}

One aim of the study was to challenge the preferred model of the previous study (Andonov et al., 2007) defined as model 1:

$$
y_{i j k l}=Y S_{i}+D I M 3_{j}+F T D_{k}+a_{l}+p e_{l}+e_{i j k l},
$$

where $y_{i j k l}$ is DMY, FC, PC, or LC for goat $l$ within YS class $i$, stage of lactation $j$, and $\mathrm{FTD} k ; Y S_{i}$ is fixed YS effect, with 42, 41, 41, and 36 classes for DMY, FC, PC and LC, respectively; $D I M 3_{j}$ is a fixed effect of stage of lactation, defined in 3-d intervals starting from d 5 , with 101 classes; $F T D_{k}$ is the random effect of FTD $k$, whereas the random animal additive genetic effect of goat $l$ is $a_{l}$; and $p e_{l}$ and $e_{i j k l}$ are random effects of permanent environment of animal and residual, respectively.

The competing model had ASR across YS (model 2):

$$
\begin{gathered}
y_{i k l}=Y S_{i}+b_{1}(\mathrm{DIM} / 305)+b_{2}(\mathrm{DIM} / 305)^{2} \\
+b_{3} \ln (305 / \mathrm{DIM})+b_{4}[\ln (305 / \mathrm{DIM})]^{2} \\
+F T D_{k}+a_{l}+p e_{l}+e_{i k l}
\end{gathered}
$$

where additional notation relative to that described for model 1 is $b_{1}, b_{2}, b_{3}$, and $b_{4}$, which are fixed ASR coefficients across YS classes, and DIM, which is day of lactation (5 to 305).

Finally, another specification capable of accounting for time trends was considered by omitting YS in model 1 and rather considering the effect of the $m$ th FY as a fixed effect $\left(F Y_{m}\right)$, otherwise for comparable notation (model 3):

$$
y_{j k l m}=D I M 3_{j}+F Y_{m}+F T D_{k}+a_{l}+p e_{l}+e_{j k l m} .
$$

As DMY was recorded more frequently than milk content in a flock, the number of observations per level of FY was higher for DMY than for FC, PC and LC $(155,146,146$ and 134 classes, with average number of records $73.8,40.4,40.4$ and 42.4 , respectively).

\section{RR Models}

The best (see Results and Discussion section) REP model (model 1) was extended by adding Legendre polynomials of either first, second, or third order, both for the random effects of animal and the permanent environment, resulting in models denoted as RRL1, RRL2, and RRL3, respectively. The RRL models can be described as follows:

$$
\begin{aligned}
& y_{i j k l o}=Y S_{i}+D^{2} 3_{j}+F T D_{k} \\
& +\sum_{m=0}^{3} a_{l m} Z_{o m}+\sum_{m=0}^{3} p e_{l m} Z_{o m}+e_{i j k l o}
\end{aligned}
$$

where the additional notation to that in model 1 is $Z_{o m}$, the polynomial $m$ for DIM $o$, where $m=0,1,2$, or 3 , of same order for both the animal and permanent environmental effects; $a_{l m}$ is the random regression coefficient on $Z_{o m}$ for the animal genetic effect; $p e_{l m}$ is the random regression coefficient on $Z_{\text {om }}$ for the permanent environmental effect; and $e_{i j k l o}$ is the random residual.

At DIM $o$, additive genetic variance $\left(\sigma_{a_{o}}^{2}\right)$, permanent environmental variance $\left(\sigma_{p e_{o}}^{2}\right)$, and heritability $\left(h^{2}{ }_{o}\right)$ were calculated as follows: 


$$
\begin{gathered}
\sigma_{a_{o}}^{2}=\mathbf{z}_{\mathbf{o}}{ }^{\prime} \mathbf{G z}_{\mathbf{o}} ; \\
\sigma_{p e_{o}}^{2}=\mathbf{z}_{\mathbf{o}}{ }^{\prime} \mathbf{P} \mathbf{z}_{\mathbf{o}} ; \\
h_{o}^{2}=\frac{\sigma_{a_{o}}^{2}}{\sigma_{a_{o}}^{2}+\sigma_{p e_{o}}^{2}+\sigma_{e}^{2}},
\end{gathered}
$$

where $\mathbf{z}_{\mathbf{o}}$ is a vector of polynomials in the model for DIM $o, \mathbf{G}$ is the (co)variance matrix for animal $\mathrm{RR}$ coefficients, $\mathbf{P}$ is the (co)variance matrix for permanent environment $\mathrm{RR}$ coefficients, and $\sigma_{e}^{2}$ is the residual variance. Note that model 1 is equivalent to the random regression model of order $m=0$.

In all models, homogeneous residual variances were assumed. The random animal genetic effects were assumed to have (co)variance structure proportional to the additive relationship matrix, whereas the repeated animal effects were unstructured. Variance component estimations were carried out one trait at a time, for REP model with VCE version 5.1.2 software (Kovač and Groeneveld, 2003) and for RRL with DMU version 6 software (Madsen and Jensen, 2002). Predictions of breeding values were done with PEST version 4.2.1 software (Groeneveld, 1990).

\section{Model Comparison}

Models were compared for their fit and for the complete model's ability to predict excluded observa- tions through calculation of the mean-squared error of prediction (MSEP) or through correlating predicted breeding values based on relatives only with the observed phenotype of animal itself. Here, these 3 approaches were used to choose the complexity of random effects models.

MSEP. Models were compared by use of MSEP calculated by randomly excluding $5 \%$ of the observations, but always keeping at least 1 record per animal. These excluded observations were predicted from the remaining data on the basis of the relevant model. Six different subsamples were made, and predictive ability of a model was calculated as the average over the subsamples according to the following:

$$
\operatorname{MSEP}=\left[\sum_{i=1}^{6}\left(\frac{1}{n} \sum_{j=1}^{n}\left(y_{j}-\hat{y}_{j}\right)^{2}\right)\right] / 6,
$$

where $y_{j}$ is an observation $j$ and $\hat{y}_{j}$ is the predicted value of that observation, out of $n$ excluded observations. Variance components were estimated using the same model for the entire data set. In the prediction of excluded observations, these variance component estimates were used as true parameters. Additionally, model 1 and 2 were compared by calculating average MSEP over 5 to 100 DIM, 101 to 200 DIM, and 201 to 305 DIM.

Model Fit. The RRL models were also compared using MSEP, but additionally with the likelihood-ratio test (e.g., Welham and Thompson, 1997):

\begin{tabular}{|c|c|c|c|c|c|c|}
\hline Item & $\sigma_{a}^{2}$ & $\sigma_{F T D}^{2}$ & $\sigma_{p e}^{2}$ & $\sigma_{e}^{2}$ & $\mathrm{~h}^{2}$ & MSEP \\
\hline \multicolumn{7}{|l|}{ DMY } \\
\hline Model $1^{2}$ & 0.0439 & 0.0991 & 0.0543 & 0.0701 & 0.2607 & 0.8530 \\
\hline Model 2 & 0.0433 & 0.1002 & 0.0546 & 0.0700 & 0.2579 & 0.9853 \\
\hline Model 3 & 0.0301 & 0.0802 & 0.0625 & 0.0702 & 0.1849 & 1.1378 \\
\hline \multicolumn{7}{|l|}{$\mathrm{FC}$} \\
\hline Model $1^{2}$ & 0.1080 & 0.2419 & 0.0575 & 0.2889 & 0.2376 & 0.4733 \\
\hline Model 2 & 0.1080 & 0.2457 & 0.0595 & 0.2910 & 0.2356 & 0.4902 \\
\hline Model 3 & 0.0910 & 0.2269 & 0.0682 & 0.2889 & 0.2031 & 0.5448 \\
\hline \multicolumn{7}{|l|}{$\mathrm{PC}$} \\
\hline Model $1^{2}$ & 0.0167 & 0.0197 & 0.0085 & 0.0360 & 0.2733 & 0.5890 \\
\hline Model 2 & 0.0170 & 0.0201 & 0.0084 & 0.0362 & 0.2760 & 0.6352 \\
\hline Model 3 & 0.0160 & 0.0201 & 0.0091 & 0.0360 & 0.2619 & 0.6756 \\
\hline \multicolumn{7}{|l|}{ LC } \\
\hline Model $1^{2}$ & 0.0095 & 0.0085 & 0.0081 & 0.0170 & 0.2746 & 0.3363 \\
\hline Model 2 & 0.0094 & 0.0087 & 0.0082 & 0.0170 & 0.2746 & 0.3827 \\
\hline Model 3 & 0.0099 & 0.0078 & 0.0075 & 0.0170 & 0.2878 & 0.5378 \\
\hline
\end{tabular}

Table 1. Estimated additive genetic $\left(\sigma_{a}^{2}\right)$, flock test day $\left(\sigma_{F T D}^{2}\right)$, permanent environmental $\left(\sigma_{p e}^{2}\right)$, and residual $\left(\sigma_{e}^{2}\right)$ variances as well as heritability ${ }^{1}$ and mean-squared error of prediction (MSEP) for daily milk yield (DMY), fat content (FC), protein content (PC), and lactose content (LC) from 3 repeatability test-day models 
DMY

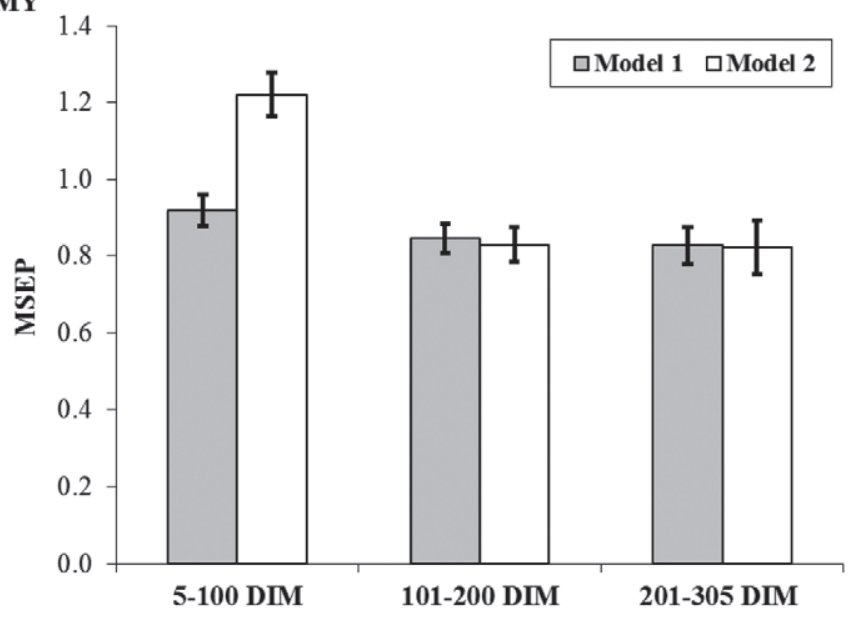

PC

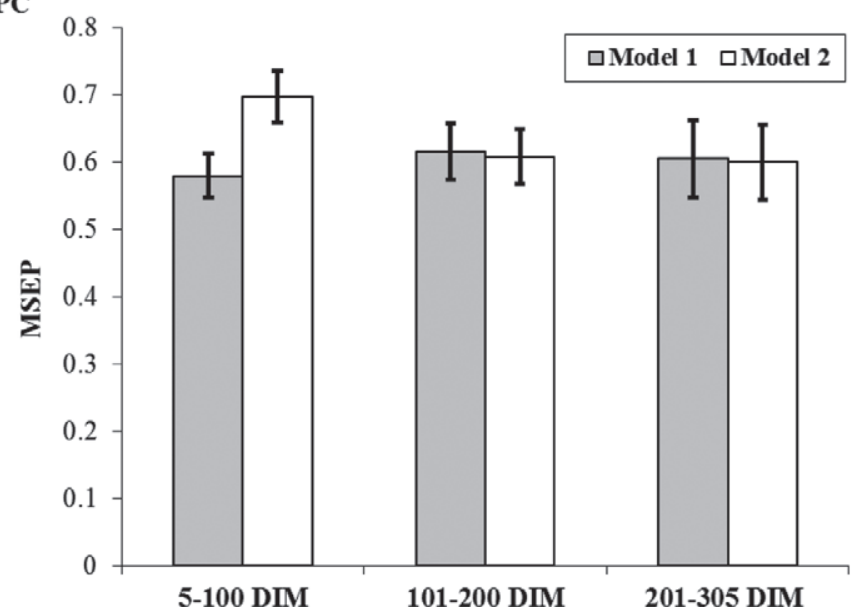

FC

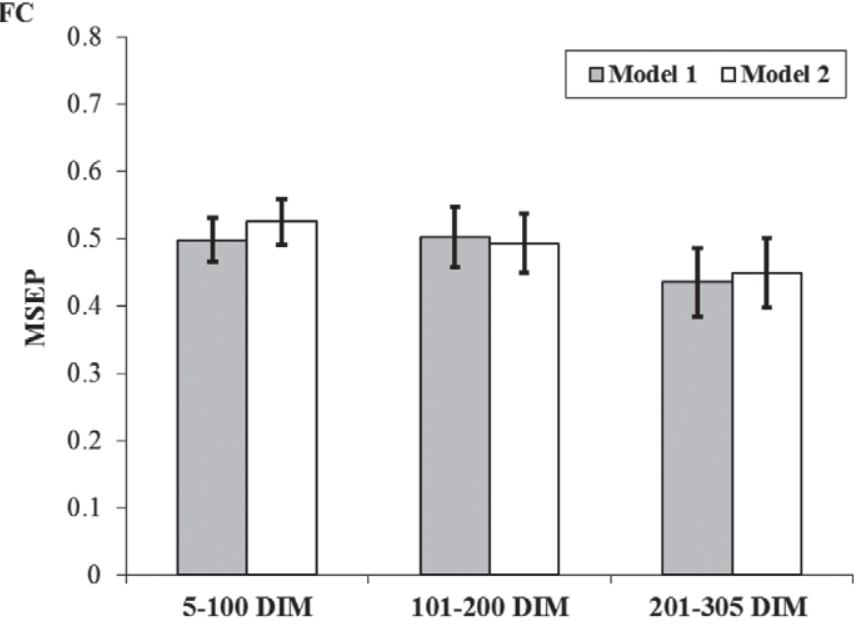

LC

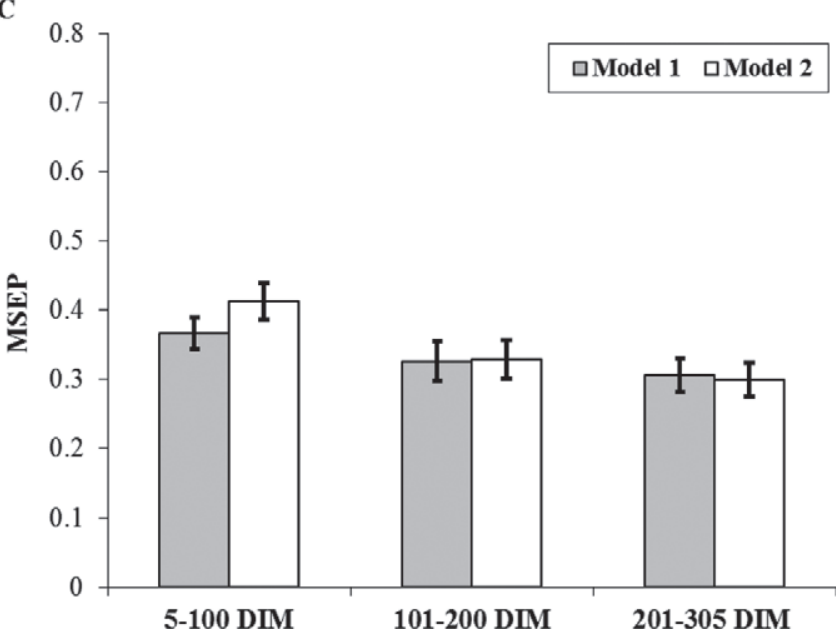

Figure 1. Average mean-squared error of prediction (MSEP) with standard error for models 1 and 2 for daily milk yield (DMY), fat content (FC), protein content (PC), and lactose content (LC), from 5 to 100 DIM, 101 to 200 DIM, and from 201 to 305 DIM.

$$
-2 \operatorname{lnRL}=-2 \ln \left(L_{(0)} / L_{(i)}\right),
$$

where $L_{(0)}$ is the restricted likelihood (RL) for the null model and $L_{(i)}$ is the restricted likelihood for the alternative model; $-2 \operatorname{lnRL}$ can only be used for comparison when the fixed effects are identical, because we base it on the restricted likelihood. Additionally, likelihood criteria penalizing for the number of parameters in the model were used: Akaike information criterion (AIC; Akaike, 1973) and the Bayesian information criterion (BIC; Schwarz, 1978), defined as follows:

$$
\mathrm{AIC}=2\left[\ln \frac{L_{(i)}}{L_{(0)}}-\left(v_{i}-v_{0}\right)\right] ;
$$

$$
\mathrm{BIC}=2\left(\ln \frac{L_{(i)}}{L_{(0)}}\right)-\left(v_{i}-v_{0}\right) \times \ln [n-r(X)],
$$

where $v_{i}$ is the number of parameters in the nested alternative model, relative to that in model 1 , taken as the null model with $v_{0}$ parameters; $n$ is the number of records; and $r(X)$ is the rank of the fixed effects incidence matrix. With this definition of the likelihoodbased criteria, models with the largest values had the best fit (Ødegård et al., 2003).

Predictive Ability. Additionally, to predict excluded phenotypes the data was split into 2 on a flock basis, keeping number of records as equal as possible (49.2\% in one data set and the remainder in the other). In both 
Table 2. Estimates of $-2 \ln$ restricted likelihood ( $-2 \ln R L)$, Akaike information criterion (AIC), Bayesian information criterion (BIC), mean-squared error of prediction (MSEP), Pearson correlation coefficients between predicted breeding values on basis of pedigree relationship only, and excluded field performance $\left(r_{E B V^{-} Y}\right)$, residual $\left(\sigma_{e}^{2}\right)$, and flock test day $\left(\sigma_{F T D}^{2}\right)$ variances for daily milk yield (DMY), fat content (FC), protein content (PC), and lactose content (LC) from a repeatability model (model 1), and random regression models with first-, second-, or third-order Legendre polynomials (RRL1, RRL2, or RRL3) for additive genetic and permanent environmental effects

\begin{tabular}{|c|c|c|c|c|c|c|c|}
\hline Item & $-2 \ln R L$ & AIC & BIC & MSEP & $r_{E B V^{-} Y}$ & $\sigma_{e}^{2}$ & $\sigma_{F T D}^{2}$ \\
\hline \multicolumn{8}{|l|}{ DMY } \\
\hline Model 1 & 0.0 & 0.0 & 0.0 & 0.853 & 0.033 & 0.070 & 0.099 \\
\hline RRL1 & 484.0 & 476.0 & 446.6 & 0.722 & 0.130 & 0.059 & 0.094 \\
\hline RRL2 & 675.1 & 655.1 & 581.8 & 0.718 & 0.162 & 0.053 & 0.093 \\
\hline RRL3 & 836.8 & 800.8 & 668.8 & 0.723 & 0.006 & 0.048 & 0.092 \\
\hline \multicolumn{8}{|l|}{$\mathrm{FC}$} \\
\hline Model 1 & 0.0 & 0.0 & 0.0 & 0.474 & 0.058 & 0.289 & 0.242 \\
\hline RRL1 & 40.6 & 32.6 & 6.0 & 0.320 & 0.134 & 0.252 & 0.242 \\
\hline RRL2 & 91.1 & 71.1 & 4.6 & 0.371 & 0.003 & 0.237 & 0.237 \\
\hline RRL3 & 84.4 & 48.4 & -71.5 & 0.409 & 0.014 & 0.231 & 0.235 \\
\hline \multicolumn{8}{|l|}{$\mathrm{PC}$} \\
\hline Model 1 & 0.0 & 0.0 & 0.0 & 0.589 & 0.101 & 0.036 & 0.020 \\
\hline RRL1 & $1,139.7$ & $1,131.7$ & $1,05.0$ & 0.512 & 0.154 & 0.024 & 0.019 \\
\hline RRL2 & 896.4 & 876.4 & 809.8 & 0.517 & 0.004 & 0.017 & 0.019 \\
\hline RRL3 & 438.8 & 402.8 & 282.9 & 0.636 & -0.001 & 0.012 & 0.020 \\
\hline \multicolumn{8}{|l|}{$\mathrm{LC}$} \\
\hline Model 1 & 0.0 & 0.0 & 0.0 & 0.337 & 0.079 & 0.017 & 0.009 \\
\hline RRL1 & 336.1 & 328.1 & 301.6 & 0.229 & 0.145 & 0.011 & 0.009 \\
\hline RRL2 & 402.7 & 382.7 & 316.5 & 0.147 & 0.023 & 0.009 & 0.008 \\
\hline RRL3 & 432.3 & 396.3 & 277.1 & 0.247 & 0.007 & 0.008 & 0.008 \\
\hline
\end{tabular}

data sets, breeding values were predicted for all animals univariately (for DMY, FC, PC, or LC) with models 1, RRL1, RRL2, or RRL3, using variance components estimated from all data. For each trait and model, the excluded phenotypes in one data set were related to their predicted breeding values in the other data set, at the relevant DIM, through calculation of the Pearson correlation coefficient $\left(r_{E B V-Y}\right)$. The expected value of this correlation $[E(r)]$ is as follows: $E(r)=k \times r_{E B V-B V}$, where $k$ is a constant, being the ratio between standard deviation of true breeding values and that of phenotype [e.g., maximum square root of heritability $\left(\mathrm{h}^{2}\right)$, when no fixed effects affect phenotypic variance]; and $r_{E B V-B V}$ is the correlation between true breeding value of the animal and the predicted (pedigree) breeding value. Note that $k$ is model independent (i.e., the same for all models). Because the breeding values were calculated from the same data sets, with only somewhat different models, the resulting breeding values were correlated. The Pearson correlation coefficients were, thus, dependent and therefore tested by the pairwise $t$-test of Dunn and Clark (1971), using DEPCOR software (Clayton Silver et al., 2006).

\section{RESULTS AND DISCUSSION}

\section{REP Models}

For the 3 REP models, MSEP results are shown in Table 1. For all traits, MSEP values were consistently in favor of model 1 , followed by model 2 , and finally model 3.

For all traits, the MSEP at the start of the lactation (5-100 DIM) was higher for model 2 than for model 1 (Figure 1), with the largest differences for DMY and PC. In mid and late lactation, MSEP for both models resembled each other. These results indicate that ASR is not sufficiently flexible in the first part of lactation when the largest change of production occurs, particularly over the first 30 DIM. Rather, the lactation should be modeled as fixed effects of DIM, as also proposed by Swalve (2000). However, with this model (model 1) and for more data, it could be relevant to specify the lactation within YS. Still, the same general result should apply: a fixed effect of group of days in lactation is more flexible than ASR (for reasonably short-day groups).

Estimated variance components (Table 1) were similar for models 1 and 2, whereas model 3 reduced the additive genetic variance, and resulted in variance components similar to those of Andonov et al. (2007) when replacing YS with FTD. So, increasing the subclass sizes to above 13 to 14 for FTD (to 40-73 for FY, and keeping FTD random as in model 3) did not release additional additive genetic variance. This is in agreement with van Bebber et al. (1997), who recommended contemporary groups modeled as fixed for subclass sizes larger or equal to 15 . 

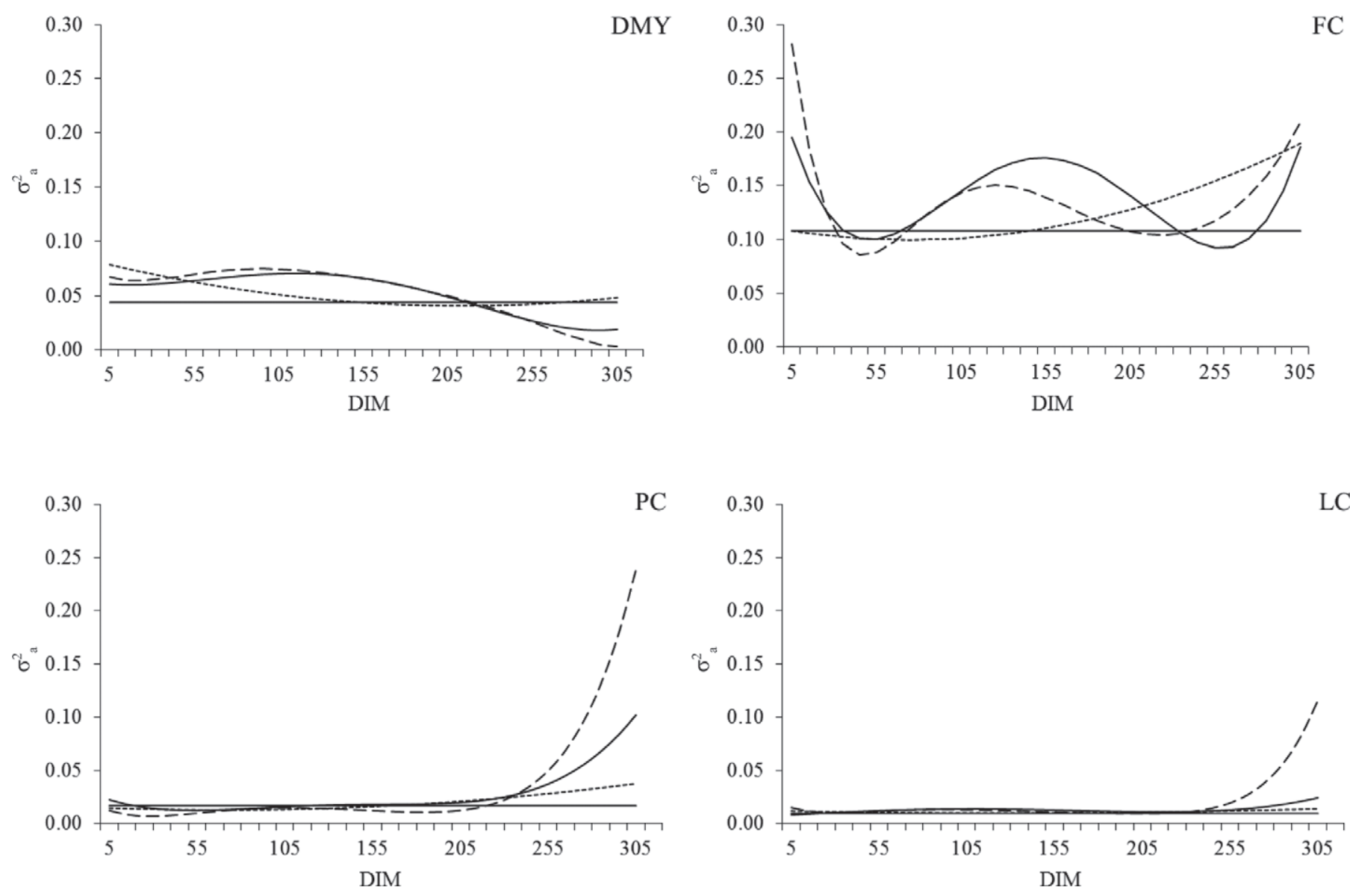

Figure 2. Estimated trajectories of animal additive genetic variance $\left(\sigma_{a}^{2}\right)$ over DIM for daily milk yield (DMY), fat content (FC), protein content (PC), and lactose content (LC) from a repeatability model (model 1, indicated with a flat line) and 3 random regression models with first- (dotted), second- (solid), or third-order (dashed) Legendre polynomials, both for the effects of additive genetic and permanent environment.

\section{RR Models}

For the REP model (model 1) and the 3 RRL models, results for model fit parameters: $-2 \operatorname{lnRL}$, AIC, BIC, MSEP, residual, as well as FTD variances are presented in Table 2. For all traits, higher orders of RR reduced the error variance estimates $\left(\sigma_{e}^{2}\right)$, as expected. For DMY, with around 5 observations per animal, the likelihood-based criteria (-2lnRL, AIC, and BIC) preferred the third-order polynomial, whereas the MSEP was in favor of a polynomial of second order. Content traits had around 3 observations per animal and for PC, all likelihood-based criteria and MSEP agreed on a first-order RR. For FC and LC smaller differences existed in likelihood values between models. Thus, BIC, due to its heavy penalizing on complexity, became more conservative in its model choice and agreed with that made by MSEP (first order for FC and second order for LC), whereas - 2lnRL or AIC preferred higher orders.
The Pearson correlation coefficients between the individual's predicted breeding values on the basis of the pedigree relationship only and excluded phenotypes are also given in Table 2. For DMY, the largest correlation was for a second-order Legendre polynomial (RRL2; significance values of $P=0.007$ for the comparison with RRL1), whereas for all 3 content traits, breeding values from RRL1 predicted excluded observation better than model $1(P=0.00003, P=0.0026$, and $P=0.0003$ for FC, PC, and LC, respectively). So, based on this cross-validation criterion, RRL2 should be preferred for DMY, and RRL1 for FC, PC, and LC.

The cross-validation criterion $\left(r_{E B V-Y}\right)$ is only proportional to the correlation between true and predicted breeding values, or accuracy of breeding values, also because data was split into two. This proportionality was used by both Gitterle et al. (2006) and Ødegård et al. (2007) to compare models, and our approach is as such related to theirs. The comparison is with respect 

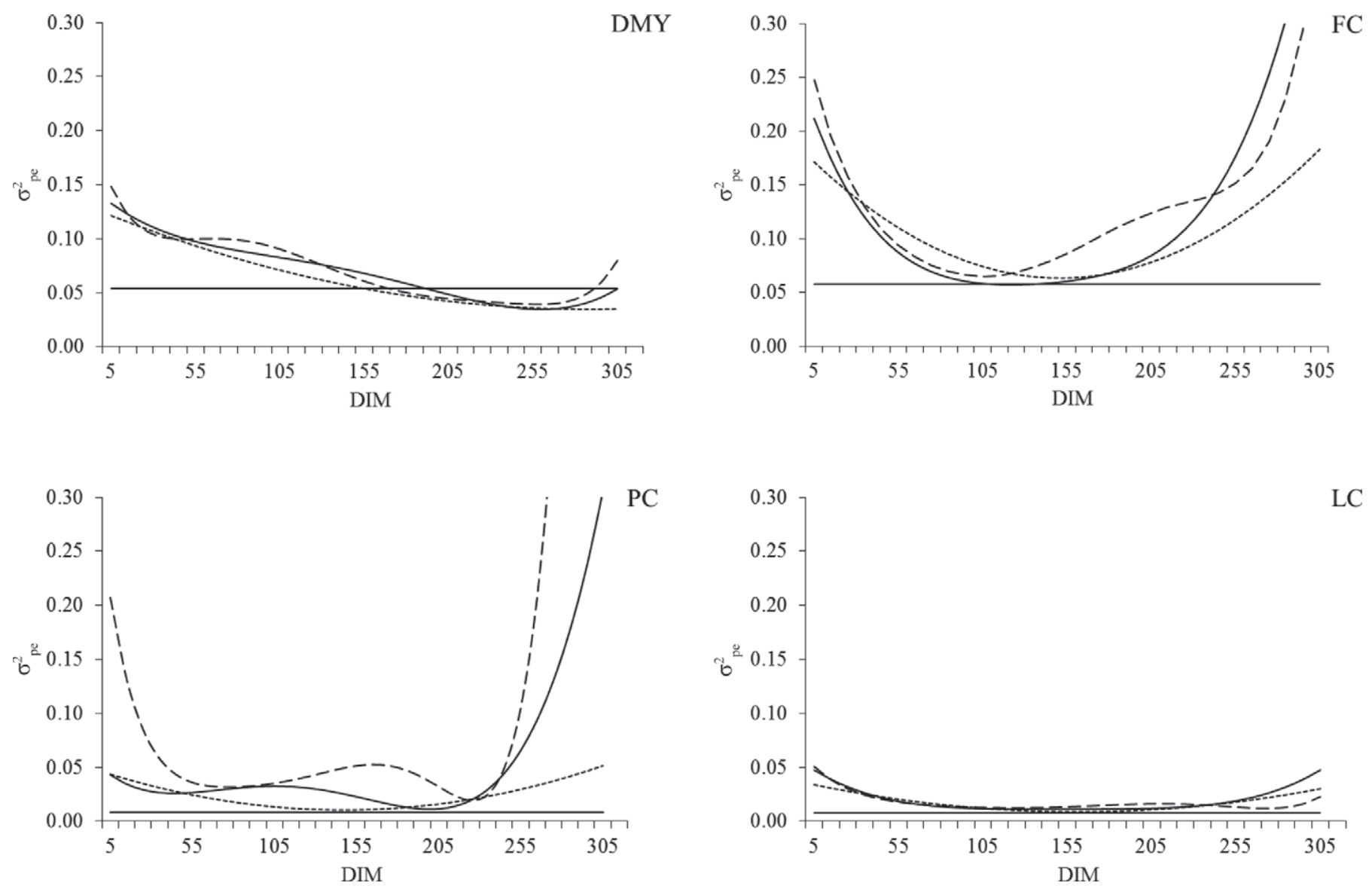

Figure 3. Estimated trajectories of permanent environmental variance $\left(\sigma_{p e}^{2}\right)$ over DIM for daily milk yield (DMY), fat content (FC), protein content (PC), and lactose content (LC) from a repeatability model (model 1, indicated with a flat line) and 3 random regression models with first- (dotted), second- (solid), or third-order (dashed) Legendre polynomials, both for the effects of additive genetic and permanent environment.

to which model produces the most genetic gain, but the advantage is only possible to derive for the data structure of the split data. One should, however, be aware that splitting may reduce connectedness and accuracy of breeding values in breeding schemes (e.g., Kennedy and Trus, 1993), especially for less well-designed data sets than those resulting from the Norwegian buck circles.

For the 4 traits, RR models always produced higher $r_{E B V-Y}$ than REP models, giving a preference to RR models in genetic evaluation based on these data. A simple pattern was revealed: for DMY a second-order RRL should be chosen, whereas for content traits, only a first-order polynomial was preferred. Other approaches based on model fitting or on MSEP produced a less-consistent pattern, giving an advantage to more complex models, although with BIC and MSEP, the results were in direction of those obtained with crossvalidation.
Estimated trajectories of the variances over the lactation for the 4 traits and the 4 models are shown in Figures 2 and 3 for the additive genetic and permanent environmental effects, respectively. For DMY, rather similar variance components were estimated with the different models, the largest in early and mid lactation, as also obtained by Zumbach et al. (2008) and Menéndez-Buxadera et al. (2010), with a comparable number of records per individual and RR models of second order. Similar literature estimates were not available for content traits. For FC, $-2 \ln R L$ chose a second-order polynomial that produced a more wavy pattern for the additive genetic variance than that of the preferred polynomial from cross-validation, of first order, increasing genetic variance somewhat toward the end of lactation. For PC, all criteria favored a first-order polynomial, again increasing genetic variance in late lactation. The same pattern existed for LC, where $-2 \ln R L$ preferred a third-order polynomial, 


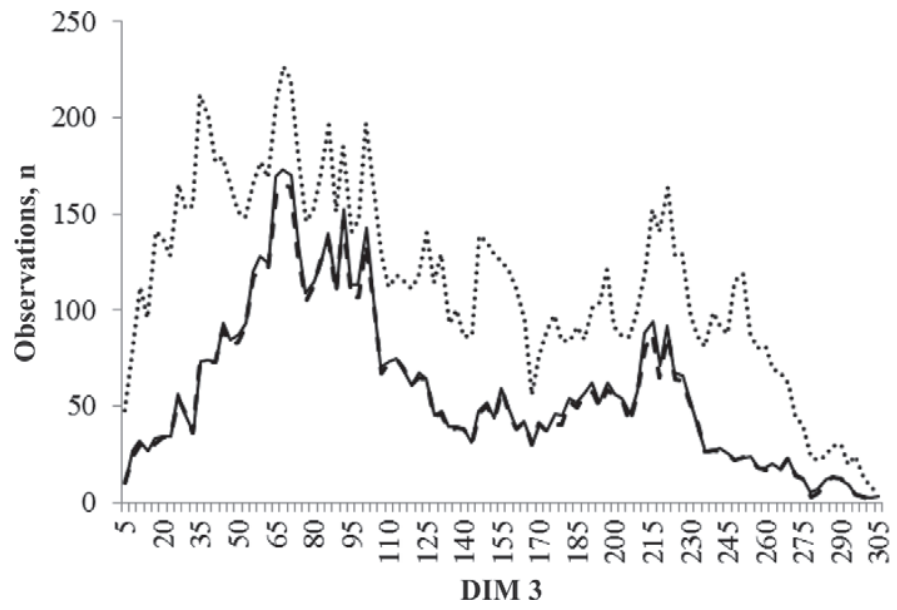

Figure 4. Number of observations (n) in classes of 3 DIM (DIM 3) for daily milk yield (DMY, dotted), fat and protein contents (FC and PC, solid), and lactose content (LC, dashed).

whereas cross-validation was in favor of a polynomial of first order. For permanent environmental variance of content traits and relevant polynomials, enlarged values were also estimated toward the end of lactation. This can result from the trajectory being most precisely determined in early and mid lactation, with most observations (Figure 4), enforcing fluctuations of the polynomial in the tails (having the least number of observations). Further, the pattern might evolve from the permanent environmental variance absorbing possible heterogeneity of the residual variance, as observed by Ødegård et al. (2003).

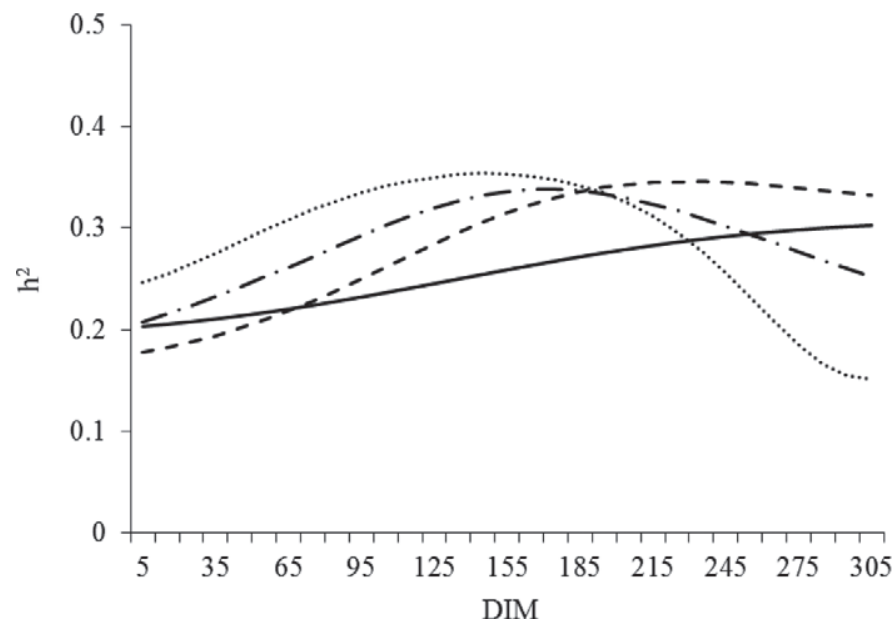

Figure 5. Estimated trajectories of heritability $\left(\mathrm{h}^{2}\right)$ over DIM for daily milk yield (DMY, dotted), fat content (FC, solid), protein content (PC, dashed), and lactose content (LC, long dash dotted) from random regression models with second-order Legendre polynomials for DMY and first-order polynomials for FC, PC, and LC, for additive genetic and permanent environmental effects.
For the preferred models from cross-validation: RRL2 for DMY and RRL1 for FC, PC, and LC, the resulting heritability estimates are depicted in Figure 5, being of considerable size, mostly above 0.2 , in accordance with industry estimates in this population, referred to by Dagnachew et al. (2011). The estimate of DMY peaked in mid lactation, somewhat in contrast to the results of Zumbach et al. (2008) and Menéndez-Buxadera et al. (2010), who found a decrease in the estimate during the course of first lactation. The corresponding estimates of the genetic correlation for DMY for different DIM are plotted in Figure 6, showing high correlation for consecutive DIM and considerably lower for days far apart $(>0.08)$. For content traits with a limited number of observations per animal, a first-order polynomial was advised, having limited flexibility. This might affect the shape of the trajectory of genetic parameters, and also that for the heritability of content traits, being higher toward the end of lactation. Another consequence of limited flexibility might be far higher genetic correlation estimates for content traits for days far apart $(>0.50$, 0.25 , and 0.56 for FC, PC, and LC, respectively) than for DMY (Figure 6).

\section{CONCLUSIONS}

In comparison between REP and RR models, a simple pattern was revealed; in Norwegian dairy goats with typically 5 observations recorded for milk yield per lactation, a second-order orthogonal polynomial should be chosen for both animal additive genetic and permanent environmental effects, whereas for content traits, with around 3 observations per goat, a first-order polynomial should have preference. This pattern resulted from cross-validation, correlating pedigree breeding values and excluded phenotypes, whereas other approaches based on model fitting or MSEP tended to favor more complex models. The correlation from cross-validation was shown proportional to the correlation between true and predicted breeding values. This implies that genetic merit was predicted most precisely with random regression, which in practice, should be adopted in a multivariate setup.

\section{ACKNOWLEDGMENTS}

The study was funded by the HERD Project financed by the Norwegian Ministry of Foreign Affairs (MFA, Oslo, Norway). Access to the data was given by the Norwegian Association of Sheep and Goat Breeders (Ås, Norway) in agreement 001.2004 who, together with the Department of International Environment and Development Studies-NORAGRIC - at the Norwegian University of Life Sciences (UMB, Ås, Norway), covered some expenses. 
DMY
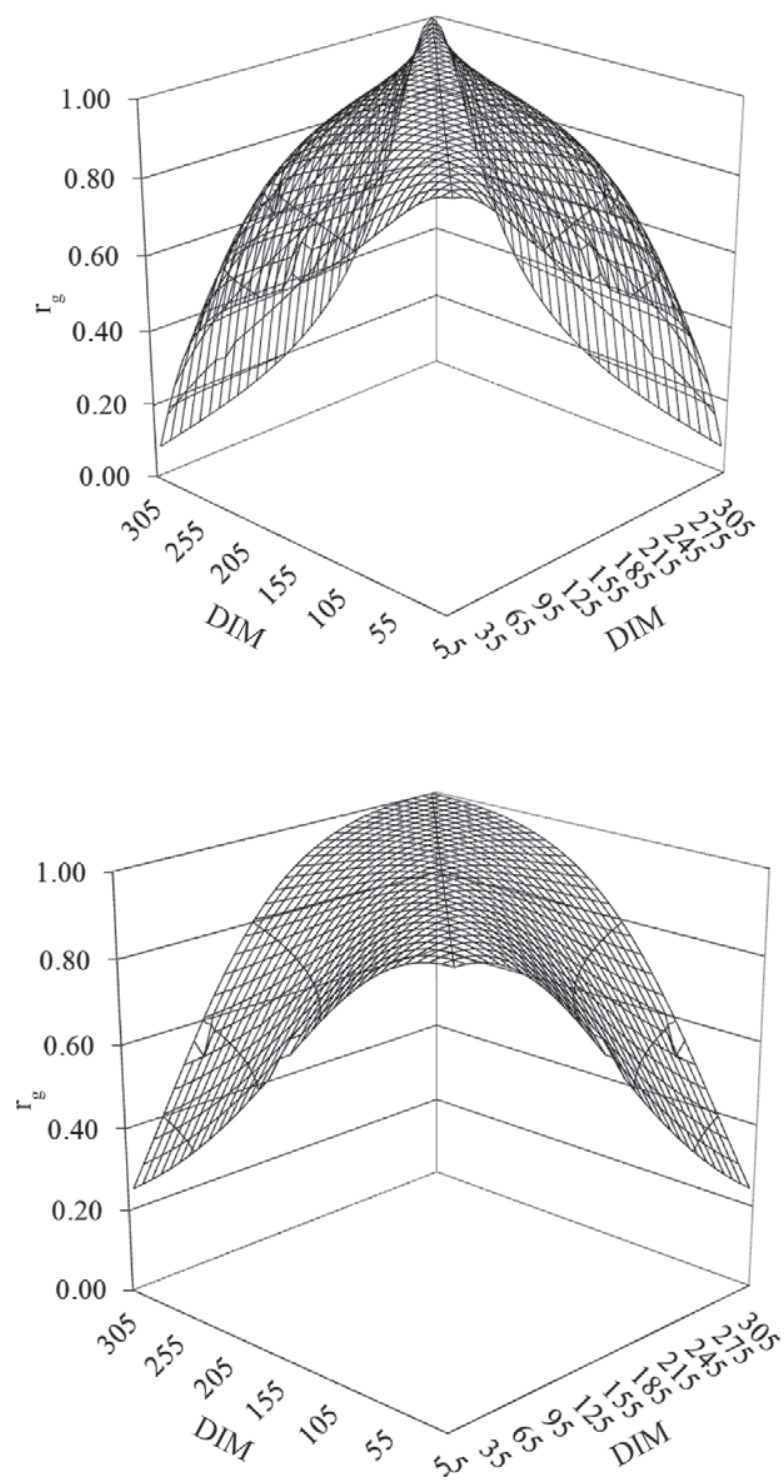

PC
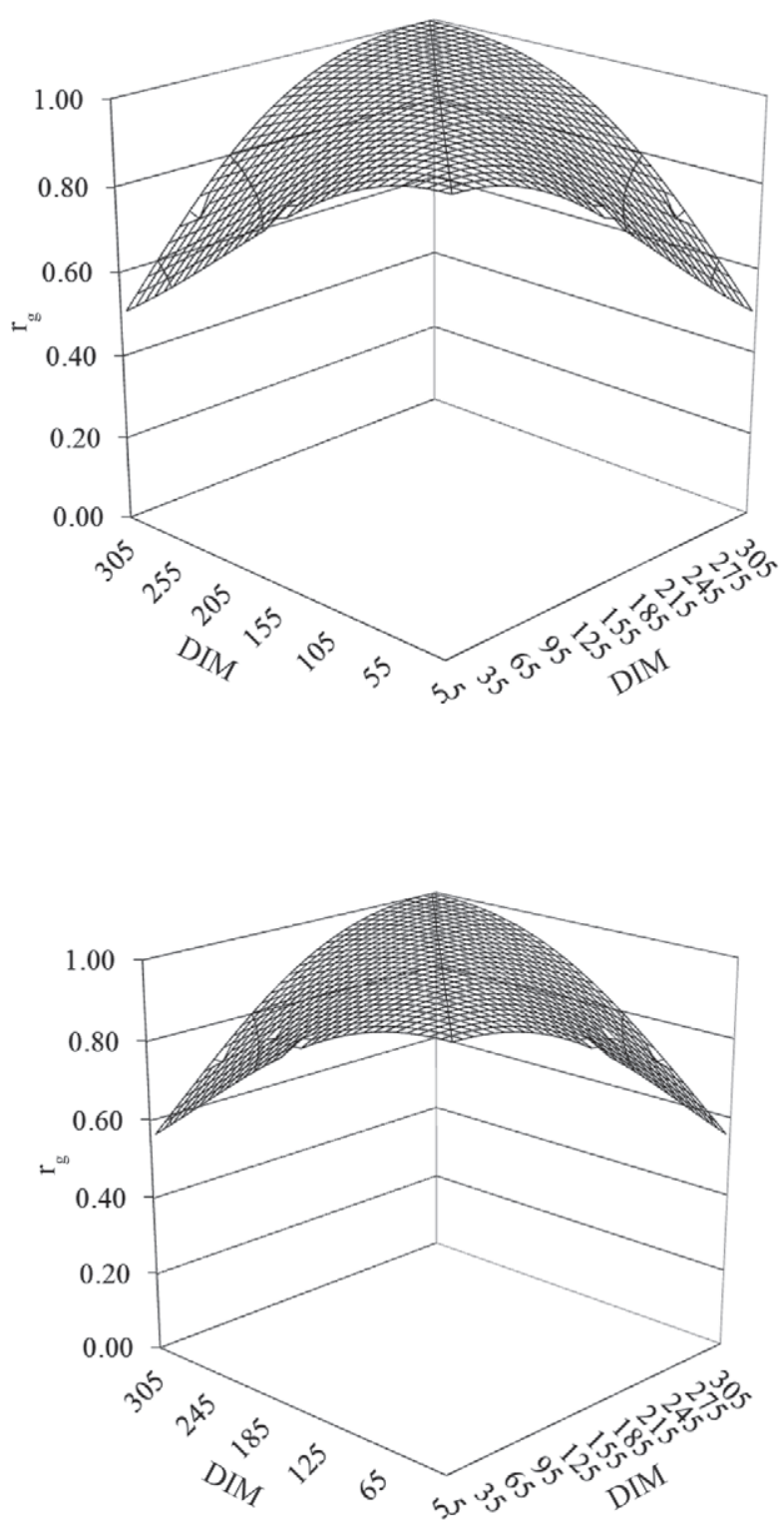

Figure 6. Estimated genetic correlations $\left(\mathrm{r}_{\mathrm{g}}\right)$ between different DIM for daily milk yield (DMY), fat content (FC), protein content (PC), and lactose content (LC) from random regression models with second-order Legendre polynomials for DMY and with first-order polynomials for FC, $\mathrm{PC}$, and $\mathrm{LC}$, for additive genetic and permanent environmental effects.

\section{REFERENCES}

Ådnøy, T., I. Nævdal, and M. Svendsen. 2000. Buck circles for dairy goat breeding in Norway. Page 1,049 in Proc. 7th Int. Conf. Goats, Tours, France. Institut de l'Elevage, Paris, France.

Akaike, H. 1973. Information theory as an extension of the maximum likelihood principle. Pages 267-281 in Proc. 2nd Int. Symp. Info Theory. B. N. Petrov and F. Csaki, ed. Akadémiai Kiadó, Budapest, Hungary.

Ali, T. E., and L. R. Schaeffer. 1987. Accounting for covariances among test-day milk yields in dairy cows. Can. J. Anim. Sci. 67:637-644. http://dx.doi.org/10.4141/cjas87-066.

Andonov, S., J. Ødegård, I. A. Boman, M. Svendsen, I. J. Holme, T. Ådnøy, V. Vukovic, and G. Klemetsdal. 2007. Validation of testday models for genetic evaluation of dairy goats in Norway. J. Dairy Sci. 90:4863-4871. http://dx.doi.org/10.3168/jds.2006-626.
Boichard, D., L. Maignel, and É. Verrier. 1997. The value of using probabilities of gene origin to measure genetic variability in a population. Genet. Sel. Evol. 29:5-23. http://dx.doi.org/10.1186/12979686-29-1-5.

Clayton Silver, N., J. B. Hittner, and K. May. 2006. A FORTRAN 77 program for comparing dependent correlations. Appl. Psychol. Meas. 30:152-153. http://dx.doi.org/10.1177/0146621605277132.

Dagnachew, B. S., G. Thaller, S. Lien, and T. Ånnøy. 2011. Casein SNP in Norwegian goats: Additive and dominance effects on milk composition and quality. Genet. Sel. Evol. 43:31-42. http:// dx.doi.org/10.1186/1297-9686-43-31.

Dunn, O. J., and V. Clark. 1971. Comparison of tests of the equality of dependent correlation coefficients. J. Am. Stat. Assoc. 66:904908.

Gitterle, T., J. Ødegård, B. Gjerde, M. Rye, and R. Salte. 2006. Genetic parameters and accuracy of selection for resistance to White 
Spot Syndrome Virus (WSSV) in Panaeus (Litopenaeus) vannnamei using different statistical models. Aquaculture 251:210-218. http://dx.doi.org/10.1016/j.aquaculture.2005.06.008.

Groeneveld, E. 1990. PEST User's Manual. Institute for Animal Science (FAL), Neustadt, Germany.

Kennedy, B. W., and D. Trus. 1993. Considerations on genetic connectedness between management units under an animal model. J. Anim. Sci. 71:2341-2352.

Kirkpatrick, M., and N. Heckman. 1989. A quantitative genetic model for growth, shape, reaction norms, and other infinite-dimensional characteristics. J. Math. Biol. 27:429-450.

Kovač, M., and E. Groeneveld. 2003. VCE 5 User's Guide and Reference Manual. University of Ljubljana, Slovenia.

Madsen, P., and J. Jensen. 2002. A User's Guide to DMU—A Package for Analyzing Multivariate Mixed Models. Version 6, release 4.4. Danish Inst. Agric. Sci., Tjele, Denmark.

Menéndez-Buxadera, A., A. Molina, F. Arrebola, M. J. Gil, and J. M. Serradilla. 2010. Random regression analysis of milk yield and milk composition in the first and second lactations of Murciano-Granadina goats. J. Dairy Sci. 93:2718-2726. http://dx.doi. org/10.3168/jds.2009-2571.

Nævdal, I., Å. Lohn, V. Fagerland, T. Ådnøy, H. Paulenz, and E. Wallin. 2005. Fagrapport 2004. Geitavl. Avlsrådet for geit. Fagforum geit. NSG. (In Norwegian).

Ødegård, J., J. Jensen, G. Klemetsdal, P. Madsen, and B. Heringstad. 2003. Genetic analysis of somatic cell score in Norwegian cattle using random regression test-day models. J. Dairy Sci. 86:41034114. http://dx.doi.org/10.3168/jds.S0022-0302(03)74024-7.

Ødegård, J., I. Olesen, B. Gjerde, and G. Klemetsdal. 2007. Evaluation of statistical models for genetic analysis of challenge-test data on ISA resistance in Atlantic salmon (Salmo salar): Prediction of progeny survival. Aquaculture 266:70-76. http://dx.doi. org/10.1016/j.aquaculture.2007.02.012.

Schwarz, G. 1978. Estimating the dimension of a model. Ann. Stat. 6:461-464. http://dx.doi.org/10.1214/aos/1176344136.

Swalve, H. H. 2000. Theoretical basis and computational methods for different test-day genetic evaluation methods. J. Dairy Sci. $\quad 83: 1115-1124 . \quad$ http://dx.doi.org/10.3168/jds.S00220302(00)74977-0.

van Bebber, J., N. Reinsch, W. Junge, and E. Kalm. 1997. Accounting for herd, year and season effects in genetic evaluations of dairy cattle: A review. Livest. Prod. Sci. 51:191-203. http://dx.doi. org/10.1016/S0301-6226(97)00058-4.

Welham, S. J., and R. Thompson. 1997. Likelihood ratio tests for fixed model terms using residual maximum likelihood. J. R. Stat. Soc., B 59:701-714. http://dx.doi.org/10.1111/1467-9868.00092.

Zumbach, B., S. Tsuruta, I. Misztal, and K. J. Peters. 2008. Use of a test day model for dairy goat milk yield across lactations in Germany. J. Anim. Breed. Genet. 125:160-167. http://dx.doi. org/10.1111/j.1439-0388.2007.00718.x. 